\title{
Amphiregulin and Epiregulin mRNA expression in primary colorectal cancer and corresponding liver metastases
}

Hidekazu Kuramochii*, Go Nakajima', Yuka Kaneko², Ayako Nakamura², Yuji Inoue ${ }^{2}$, Masakazu Yamamoto² and Kazuhiko Hayashi ${ }^{1}$

\begin{abstract}
Background: Amphiregulin (AREG) and Epiregulin (EREG), ligands of EGFR, are reported to be predictive biomarkers of colorectal cancer patients treated with Cetuximab, an anti-EGFR antibody. The purpose of this study is to determine the correlation of AREG and EREG expression between primary colorectal cancer and corresponding liver metastases.
\end{abstract}

Methods: One hundred twenty colorectal cancer patients with liver metastases (100 with synchronous metastases, 20 with metachronous) were evaluated. No patients had ever received anti-EGFR antibody agents. AREG and EREG mRNA expression from both the primary tumor and liver metastases were measured using real-time RT-PCR. KRAS codon 12, 13 mutation status was analyzed by direct sequencing.

Results: Modest, but significant, correlations were observed between primary tumor and corresponding liver metastases in both AREG mRNA expression (Rs $=0.54, \mathrm{p}<0.0001$ ) and EREG mRNA expression (Rs $=0.58, \mathrm{p}<$ 0.0001). AREG and EREG mRNA expression was strongly correlated in both the primary tumor (Rs $=0.81, \mathrm{p}<$ 0.0001 ) and the liver metastases ( $R s=0.87, p<0.0001)$. No significant survival difference was observed between low and high AREG or EREG patients when all 120 patients were analyzed. However, when divided by KRAS status, KRAS wild-type patients with low EREG mRNA levels in the primary site showed significantly better overall survival rates than those with high levels $(p=0.018)$. In multivariate analysis, low EREG expression was significantly associated with better overall survival $(p=0.006)$.

Conclusions: AREG and EREG expression showed a modest correlation between primary tumor and liver metastases. As EREG mRNA expression was associated with decreased survival, it is appeared to be a useful prognostic marker in KRAS wild-type patients who never received anti-EGFR therapy.

\section{Background}

Epidermal growth factor receptor (EGFR) is known to be involved in signaling pathways affecting cellular growth, differentiation, and proliferation [1]. To block the activation of this receptor, the anti-EGFR antibody agents Cetuximab and Panitumumab have been developed, and offer promising results for cases of metastatic colorectal cancer (CRC) [2-4]. Recently, several clinical trials demonstrated that somatic mutations in KRAS are

\footnotetext{
* Correspondence: Kuramochi@chemo.twmu.ac.jp

'Department of Chemotherapy and Palliative Care, Tokyo Women's Medical

University, 8-1 Kawadacho, Shinjukuku, Tokyo, Japan Full list of author information is available at the end of the article
}

associated with a lack of sensitivity to anti-EGFR antibody agents [5-7], suggesting that KRAS is a definite predictive biomarker for anti-EGFR antibody. However, even in patients with KRAS wild-type tumors, the response rates are between 10 and 40\% [8]. Thus, other biomarkers are required for predicting which patients will benefit from anti-EGFR antibody therapy.

Amphiregulin (AREG) and Epiregulin (EREG) belong to the epidermal growth factor (EGF) family, and act as mitogenic stimulators through binding to EGFRs [9]. Recently, two large studies on AREG and EREG expression in patients with colorectal cancer who received Cetuximab were published. Khambata-Ford et al.
C Biomed Central 
analyzed the gene expression profile of 110 patients with colorectal cancer treated with Cetuximab to identify genes that were expressed differentially between the disease control group and the non-responders, demonstrated that AREG and EREG expression was associated with progression-free survival [10]. In this study, samples were collected from the metastatic site. Jacobs et al. examined 220 colorectal cancer patients treated with Cetuximab, and reported that there was a significant association between EREG and AREG expression and the response to Cetuximab in KRAS wild-type patients, but not in KRAS mutant patients [11]. Formalin-fixed paraffin-embedded (FFPE) samples from primary colorectal cancer were used in this study.

These two studies may indicate that AREG and EREG expression, as well as KRAS status acts as a predominant biomarker of sensitivity/resistance to Cetuximab. However, although the samples were taken from different sites in these two studies, there has been no previous study that has shown the relation between the gene expressions of AREG and of EREG between the primary site and a liver metastatic site. There has not as yet been clear that the gene expression from either primary or metastatic site is more associated with patients' prognosis. In addition, although these two independent studies showed the efficacy of AREG and EREG as predictive markers for Cetuximab, the clinical importance of these genes for patients who have not received antiEGFR therapy is not clear. These genes are also reported to be associated with an increased likelihood of liver metastasis $[12,13]$, which indicates that these genes may play an important role in the development of metastasis in malignancies, suggesting that they may be prognostic markers, even with patients who have never received anti-EGFR therapy.

Therefore, the aims of our study were: (1) to observe the relationship of AREG and EREG mRNA expressions between the primary site of colorectal cancer and the corresponding liver metastatic site, (2) determine whether the gene expression from the primary site or the liver metastatic site is more likely to be associated with clinical outcome, (3) determine the significance of AREG and EREG as a prognostic markers for patients who have not received anti-EGFR therapy, and see their relation to KRAS mutant status.

\section{Methods}

\section{Patients and samples}

One hundred and twenty cases of primary colorectal cancer with the corresponding liver metastases were analyzed in this study (70 men and 50 women; median age, 63.5 (range, 32- 91). The metastases in 100 patients were synchronous, and in 20 were metachronous. These patients had undergone surgical resection of primary colorectal adenocarcinoma and liver metastasis between 1998 and 2008 at the Department of Gastroenterology, Tokyo Women's Medical University, Tokyo, Japan. One hundred two patients had received fluoropyrimidinebased chemotherapy after surgery, 6 had never received chemotherapy and 12 were unknown. No patients had received neoadjuvant/adjuvant radiotherapy. No patients had ever received anti-EGFR antibody agents. All of the patients were Japanese, and all gave their written informed consent according to the institutional regulations. The characteristics of the 120 patients are shown in Table 1.

\section{Microdissection}

Formalin-fixed, paraffin-embedded tumor specimens were cut into serial sections with a thickness of $10 \mu \mathrm{m}$. For pathological diagnosis, one slide was stained with $H \& E$ and evaluated by a pathologist. Manual microdissection using a scalpel was performed if the histology was homogeneous and contained more than $90 \%$ of cancer cell tissue. For all other samples, laser-capture

Table 1 Demographic and clinical parameters of patients with metastatic colorectal cancer

\begin{tabular}{|c|c|c|}
\hline Characteristics & Frequency & $\%$ \\
\hline \multicolumn{3}{|l|}{ Age } \\
\hline Mean (range) & $63.5(32-91)$ & \\
\hline \multicolumn{3}{|l|}{ Gender } \\
\hline Male & 70 & $58.3 \%$ \\
\hline Female & 50 & $41.7 \%$ \\
\hline \multicolumn{3}{|l|}{ Anatomical Site } \\
\hline Right colon & 35 & $29.2 \%$ \\
\hline Transverse colon & 8 & $6.7 \%$ \\
\hline Left colon & 50 & $41.7 \%$ \\
\hline Rectum & 27 & $22.5 \%$ \\
\hline \multicolumn{3}{|l|}{ Histology } \\
\hline Well differentiated & 89 & $74.2 \%$ \\
\hline Moderately differentiated & 27 & $22.5 \%$ \\
\hline Poor/Mucinous & 4 & $3.3 \%$ \\
\hline \multicolumn{3}{|l|}{ Dukes Grade } \\
\hline A & 2 & $1.7 \%$ \\
\hline B & 34 & $28.3 \%$ \\
\hline C & 84 & $70.0 \%$ \\
\hline \multicolumn{3}{|l|}{ Liver synchronicity } \\
\hline Synchronous & 100 & $83.3 \%$ \\
\hline Metachronous & 20 & $16.7 \%$ \\
\hline \multicolumn{3}{|l|}{ Lymph node metastasis } \\
\hline Positive & 84 & $70.0 \%$ \\
\hline Negative & 36 & $30.0 \%$ \\
\hline \multicolumn{3}{|l|}{ Adjuvant chemotherapy } \\
\hline Received & 102 & $85.0 \%$ \\
\hline Not received & 6 & $5.0 \%$ \\
\hline Unknown & 12 & $10 \%$ \\
\hline
\end{tabular}

This study was approved by the ethics committee of Tokyo Women's Medical University, and was performed in accordance with the Declaration of Helsinki. 
microdissection (P.A.L.M. Microlaser Technologies AG, Munich, Germany) was performed to ensure that only tumor cells were dissected.

\section{RNA isolation and CDNA synthesis}

RNA isolation from formalin-fixed paraffin-embedded (FFPE) specimens was performed using an RNeasy FFPE Kit (Qiagen, Tokyo, Japan) according to the manufacturer's instructions. From the total RNA yielded, cDNA was converted using a High Capacity cDNA Reverse Transcription Kit (Applied Biosystems, Tokyo, Japan).

\section{Reverse transcription-PCR}

cDNA was pre-amplified using a Taqman PreAmp Master Mix Kit (Applied Biosystems, Tokyo, Japan) according to the manufacturer's instructions. Quantification of AREG and EREG and internal reference gene (beta-2microglobulin) was done using a fluorescence-based real-time detection method (StepOne real-time PCR system, Applied Biosystems Inc., Tokyo, Japan). Single internal reference gene (Beta-2-microglobulin) was used in this study. The primers and probes used were from Taqman Gene Expression Assays (Applied Biosystem Inc.), Assay IDs were Hs00950669_m1 for AREG, Hs00914312_m1 for EREG, and Hs99999907_m1 for beta-2-micoroglobulin. The PCR reaction mixture consisted of $10 \mu \mathrm{l}$ of Taqman Fast Universal PCR Master Mix, No UNG (Applied Biosystem Inc.), $5 \mu \mathrm{l}$ of preamplified cDNA sample, $1 \mu \mathrm{l}$ of Taqman Gene Expression Assays primers and probe $(20 \times)$, and $3 \mu \mathrm{l}$ of NucleaseFree Water. Cycling conditions were $95^{\circ} \mathrm{C}$ for $20 \mathrm{sec}-$ onds, followed by 40 cycles at $95^{\circ} \mathrm{C}$ for 1 second and $60^{\circ}$ $\mathrm{C}$ for 20 seconds. The threshold cycle (CT) value for each gene was determined by SDS software v1.2 (Applied Biosystems). Delta-CT $(\Delta \mathrm{CT})$ value, which is the difference between the CT value of the target gene and the $C T$ value of the endogenous control gene was also calculated by the same software. Delta- $\Delta C T$ $(\Delta \Delta \mathrm{CT})$, which is the difference in the $\Delta \mathrm{CT}$ value for each sample and the highest $\Delta C T$ value as a calibrator, was calculated. The $2^{-\Delta \Delta C T}$ number was used for relative mRNA quantification.

Median values were used as the cut-off values to divide high and low expression.

\section{KRAS mutation screening}

DNA was extracted from FFPE specimens using the Qiamp DNA FFPE tissue Kit (Qiagen, Tokyo, Japan) according to the manufacturer's instructions. Concentrations were measured with the ND-1000 Spectrophotometer (NanoDrop Technologies, Wilmington, DE, USA), and $500 \mu \mathrm{g}$ of DNA was added to a $6 \mu \mathrm{l}$ of forward and reverse primer and $25 \mu \mathrm{l}$ of Quick Tag HS DyeMix (Toyobo, Osaka, Japan). Primers that spanned codons 12 and 13 of the KRAS gene were: forward, $5^{\prime}-$ GAATGGTCCTGCACCAGTAA-3'; and reverse, 5'GTGTGACATGTTCTAATATAGTCA-3'. PCR cycling was run according to the followingconditions: one cycle of $94^{\circ} \mathrm{C}$ for 3 minutes, 40 cycles of $94^{\circ} \mathrm{C}$ for 30 seconds, $56^{\circ} \mathrm{C}$ for 30 seconds, and $72^{\circ} \mathrm{C}$ for 45 seconds; and one cycle of $72^{\circ} \mathrm{C}$ for 10 minutes.

PCR products were purified using the MinElute PCR Purification Kit (Qiagen). The purified PCR product was then used as a template in cycle sequencing with the Dye Terminator Cycle Sequencing (DTCS) Quick Start Kit (Beckman Coulter, Tokyo, Japan) according to the manufacturer's instructions. Nested PCR primer sequences were Forward: 5'-GTCCTGCACCAGTAATATGC; reverse: 5'-ATGTTCTAATATAGTCACATTTTC-3'. Sequencing reactions were precipitated with ethanol, and run on a CEQ-8800 Genetic Analyzer (Beckman Coulter). Direct sequencing was performed in duplicate for each sample.

\section{Statistical analysis}

The comparisons between the median mRNA levels of the primary tumor and the corresponding liver metastases, median mRNA levels of KRAS mutant and wildtype were assessed using the Wilcoxon signed-rank test. The correlation between the mRNA levels of primary tumor and of liver metastases was assessed using Spearman's rank correlation. The Kaplan-Meier method was used for survival curves, and the log-rank test was used for statistical analysis. Overall survival was defined as the time from the day of primary tumor resection to death from any cause. Multivariate analyses were performed by Cox proportional hazard model. Statistical significance was recognized at P-values of less than 0.05 . All values were two-sided.

\section{Results}

AREG and EREG relative mRNA expression levels in primary colorectal cancer and liver metastases

There were no significant differences of median AREG and EREG relative mRNA levels between primary tumor and liver metastases (AREG: primary vs. liver $=0.16$ vs. $0.21, \mathrm{p}=0.34$, EREG: 0.030 vs. $0.034, \mathrm{p}=0.057)($ Table 2$)$.

Table 2 Median Values of AREG and EREG mRNA in primary tumor and liver metastases

\begin{tabular}{llll}
\hline & Primary tumor & Liver metastases & P-value \\
\hline AREG (median) & 0.16 & 0.21 & 0.34 \\
(range) & $(0.00-3.74)$ & $(0.00-3.67)$ & N.S. \\
EREG (median) & 0.030 & 0.034 & 0.057 \\
(range) & $(0.00-0.256)$ & $(0.00-0.419)$ & N.S. \\
\hline
\end{tabular}




\section{Correlation of AREG and EREG expression between primary tumor and liver metastases}

Modest, but significant correlation was seen with respect to AREG mRNA expression between primary tumor and corresponding liver metastases $(\mathrm{Rs}=0.57, \mathrm{p}<0.0001$ ) (Figure 1a). The same results were observed in EREG mRNA expression $(\mathrm{Rs}=0.53, \mathrm{p}<0.0001)$ (Figure $1 \mathrm{~b}$ ). Regarding AREG expression, the correlation coefficient (Rs) was 0.357 in the patients with synchronous metastases, and 0.60 in those with metachronous metastases. Regarding EREG expression, the correlation coefficient (Rs) was 0.58 in the patients with synchronous metastases, and 0.36 in those with metachronous metastases.

\section{Correlation of expression levels between AREG and EREG}

Strong correlations were observed between AREG mRNA expression and EREG mRNA expression in both the primary site $(\mathrm{Rs}=0.82, \mathrm{p}<0.0001)$ (Figure $2 \mathrm{a})$, and in the liver metastatic sites $(\mathrm{Rs}=0.87, \mathrm{p}<0.0001)$ (Figure $2 \mathrm{~b}$ ).

\section{AREG and EREG expression and KRAS status}

KRAS status was evaluated in 110 patients. Sixty-five (59\%) patients showed the KRAS wild-type, and 45 (41\%) showed the mutant type. No differences of median AREG or EREG mRNA levels were observed between KRAS wild-type and mutant types in the primary site (AREG: wild-type vs. mutant $=0.15$ vs. 0.17 ; $\mathrm{p}$ $=0.40$, EREG: wild-type vs. mutant $=0.027$ vs $0.030 ; \mathrm{p}$ $=0.45$ ) or the liver metastatic site (AREG: 0.18 vs. 0.17 ; $\mathrm{p}=0.86$, EREG: 0.043 vs. $0.035 ; \mathrm{p}=0.73$ ).

\section{AREG and EREG expression and overall survival by KRAS status}

There was no difference in overall survival between the patients with KRAS wild-type and those with mutant type $(\mathrm{p}=0.71)$. When all 120 patients were analyzed, there were no survival differences between the patients with high AREG expression and those with low expression $(p=0.92)$, or between the patients with high EREG expression and those with low expression $(\mathrm{p}=0.84)$ in the primary site. However, after division by KRAS status, the patients with low EREG expression in the primary tumor had significantly better overall survival than those with high expression in the KRAS wild-type group ( $\mathrm{p}=$ 0.018 , median survival time: 2222 days vs. 1190 days) (Figure 3a). The opposite result was observed in the KRAS mutant group; the patients with high EREG expression in primary tumor had better survival than those with low expression ( $\mathrm{p}=0.045$, median survival time: high vs. low = 1743 days vs 1113 days) (Figure $3 \mathrm{~b})$. With respect to AREG expression in primary site, no statistical differences were observed in overall survival between the patients with low AREG expression and those with high expression either among the KRAS wild-type group ( $\mathrm{p}=0.16$ ) or among the KRAS mutant group $(\mathrm{p}=0.18)$. The relative mRNA expression levels in the liver metastatic sites did not correlate with overall survival time either among the KRAS wild-type group (AREG, $p=0.26$; EREG: $p=0.28$ ), or among the KRAS mutant group (AREG: $\mathrm{p}=0.81$, EREG: $\mathrm{p}=0.63$ ).

In multivariate analysis, low EREG expression in the primary tumor site was significantly related to better overall survival $(\mathrm{p}=0.006)$ in KRAS wild-type patient group, as well as lymph node metastasis $(\mathrm{p}=0.014)$ (Table 3)

\section{Discussion}

In this study, modest correlations of AREG and EREG relative mRNA expression were observed between primary colorectal cancer and corresponding liver metastases. We
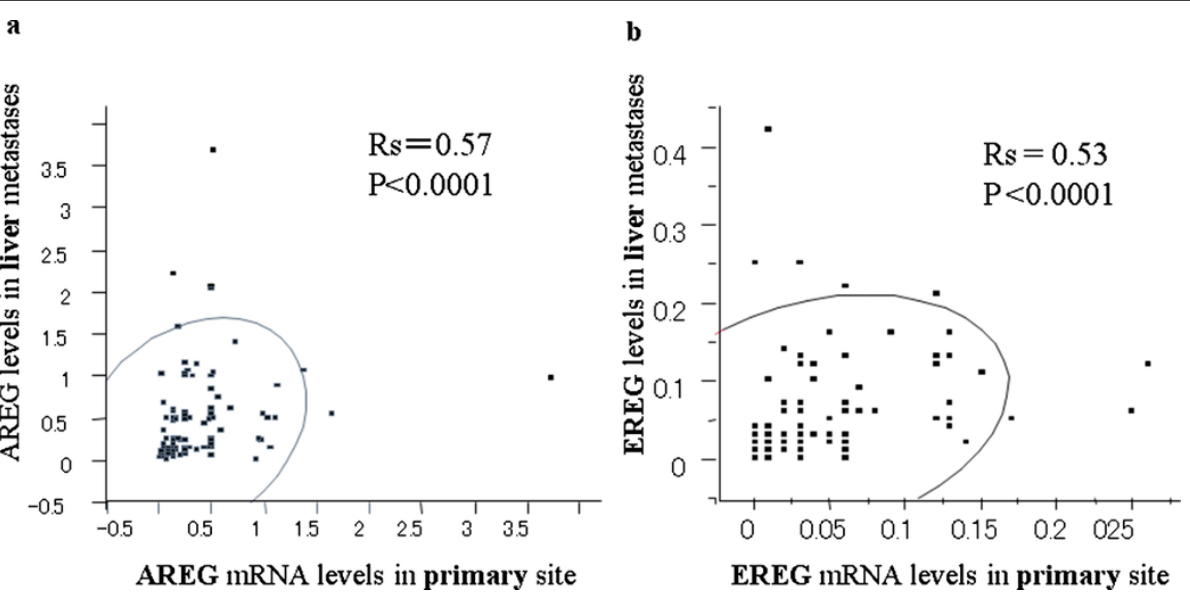

Figure 1 Correlation of mRNA expression between primary colorectal cancer and corresponding liver metastases. Figure 1-a: AREG mRNA expression; correlation coefficient (Rs) $=0.57, p<0.0001$. Figure 1-b: EREG mRNA expression; $R s=0.53, p<0.0001$. 

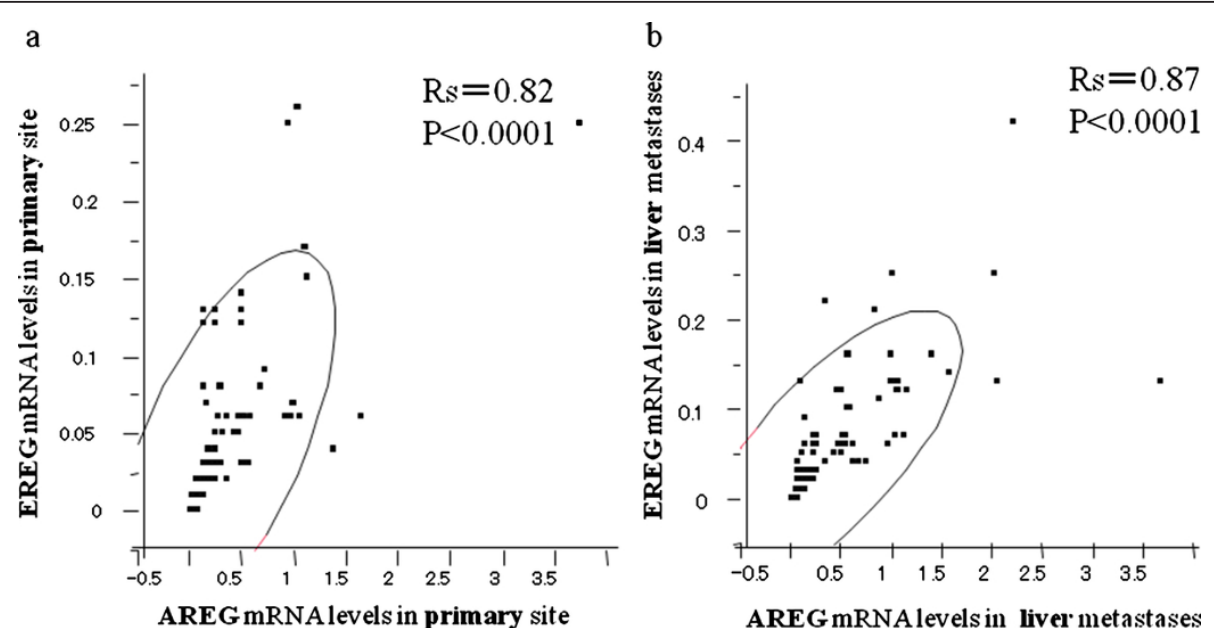

Figure 2 Correlation of mRNA expression between AREG and EREG in primary colorectal cancer (2-a) and liver metastases (2-b). Figure 2-a: primary site; correlation coefficient $(\mathrm{Rs})=0.82, \mathrm{p}<0.0001$. Figure 2 -b: metastatic site; $\mathrm{Rs}=0.87, \mathrm{p}<0.0001$.

have previously reported the correlation of VEGF [14], EGFR [15], and 5-FU metabolism-related genes [16] between primary colorectal tumor and liver metastases. Although EGFR mRNA expression showed a relatively strong correlation between the primary tumor and metastases [15], the correlations of AREG and EREG, which are the ligands of the EGFR family, between primary and metastases were weaker than that. The median values of AREG and EREG expression did not differ between primary cancer and metastases, which suggested that there was no up-regulation in the liver metastases. The strength of correlation was similar between synchronous and metachronous metastases, which suggested that expression levels were well preserved, even in relapse, for long time after primary tumor resection. Interestingly, the significance of EREG as a prognostic biomarker, which was observed in this study, was seen only on expression from the primary tumor, not on expression from the liver metastases. This data suggests that the expression from primary tumor reflects biological malignancy, and this relationship weakens in metastatic lesions after various biological modifications in the process of metastasis.

In this study, low EREG expression levels from primary tumors were significantly associated with overall survival only among patients with KRAS wild-type status. Although several previous reports demonstrated the association between EREG (and/or AREG) expression and survival time in KRAS wild-type patients who had received Cetuximab [10,11,17-19], no previous paper reported the association in colorectal cancer patients who had never received anti-EGFR antibody. The reason that this association was observed only in KRAS wild-

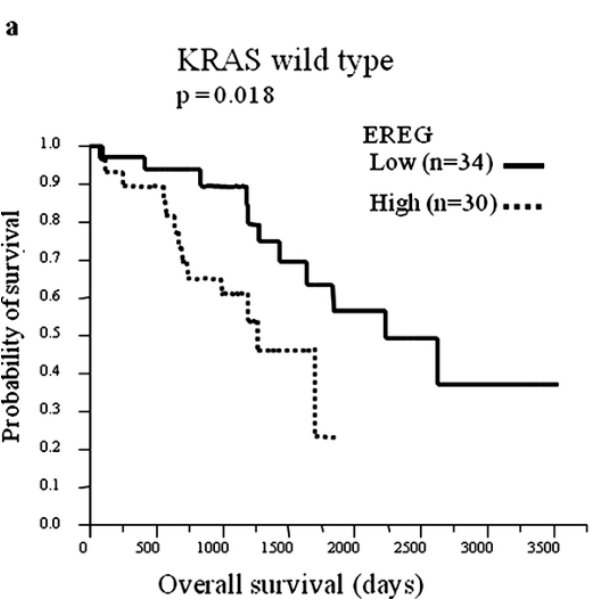

b

KRAS mutant type $\mathrm{p}=0.045$

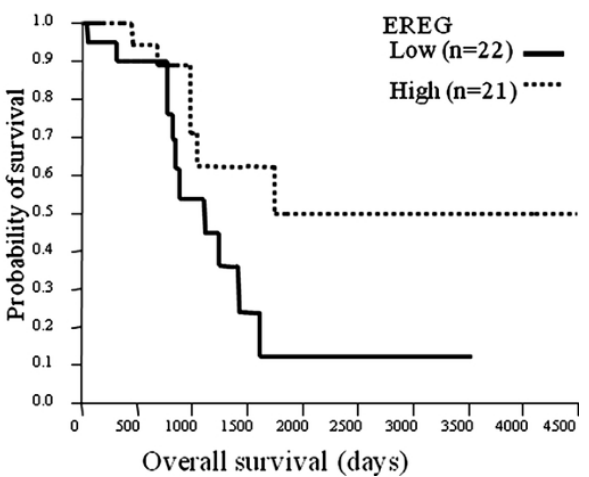

Figure 3 Kaplan-Meier Curve of overall survival by EREG mRNA expression in KRAS wild-type group (3-a) and KRAS mutant group (3b). Figure 3-a: KRAS wild-type group ( $p=0.018$, median survival; low vs high $=2222$ vs 1190 days). Figure 3-b: KRAS mutant group ( $p=0.045$; median survival; low vs high $=1113$ vs 1743 days). Cut-off line: median value of EREG expression levels. 
Table 3 Multivariate analysis (Cox proportional hazard model)

\begin{tabular}{|c|c|c|c|c|c|c|c|}
\hline \multicolumn{8}{|c|}{ KRAS Wild-type patients $(n=66)$} \\
\hline \multirow[t]{2}{*}{ Factor } & \multirow[t]{2}{*}{ Cut-off } & \multirow[t]{2}{*}{ No. of pts } & \multirow[t]{2}{*}{ Median times (months) } & \multicolumn{2}{|c|}{ Univariate analysis } & \multicolumn{2}{|c|}{ Multivariate analysis } \\
\hline & & & & $\mathrm{HR}(95 \% \mathrm{Cl})$ & p-valule & $\mathrm{HR}(95 \% \mathrm{Cl})$ & p-value \\
\hline \multirow[t]{3}{*}{ EREG } & Low & 34 & 74.1 & 1.00 & $0.021^{*}$ & 1.00 & $0.006^{*}$ \\
\hline & High & 30 & 42.1 & 2.80 & & 3.73 & \\
\hline & & & & $(1.17-7.03)$ & & $(1.46-10.10)$ & \\
\hline \multirow[t]{3}{*}{$\mathrm{pN}$} & Negative & 15 & not reached & 1.00 & $0.022^{*}$ & 1.00 & $0.014^{*}$ \\
\hline & Positive & 49 & 47.6 & 3.28 & & 3.66 & \\
\hline & & & & $(1.17-11.74)$ & & $(1.28-13.34)$ & \\
\hline \multirow[t]{3}{*}{ Depth } & $\mathrm{T} 1,2$ & 8 & not reached & 1.00 & 0.398 & 1.00 & 0.111 \\
\hline & $\mathrm{T} 3,4$ & 56 & 56.6 & 1.78 & & 2.98 & \\
\hline & & & & $(0.52-11.16)$ & & $(0.80-19.41)$ & \\
\hline \multicolumn{8}{|c|}{ KRAS mutant patients $(n=43)$} \\
\hline \multirow[t]{2}{*}{ Factor } & Cut-off & No. of pts & Median times (months) & Univ & analysis & Multi & analysis \\
\hline & & & & HR $(95 \% \mathrm{Cl})$ & p-valule & $\mathrm{HR}(95 \% \mathrm{Cl})$ & p-value \\
\hline \multirow[t]{3}{*}{ EREG } & Low & 22 & 37.1 & 1.00 & $0.048^{*}$ & 1.00 & 0.090 \\
\hline & High & 21 & 58.1 & 0.37 & & 0.42 & \\
\hline & & & & $(0.13-0.99)$ & & $(0.14-1.14)$ & \\
\hline \multirow[t]{3}{*}{$\mathrm{pN}$} & Negative & 17 & not reached & 1.00 & 0.182 & 1.00 & 0.383 \\
\hline & Positive & 26 & 41.4 & 1.98 & & 1.58 & \\
\hline & & & & $(0.73-6.25)$ & & $(0.58-5.03)$ & \\
\hline \multirow[t]{3}{*}{ Depth } & $\mathrm{T} 1,2$ & 6 & not reached & 1.00 & 0.178 & 1.00 & 0.301 \\
\hline & $\mathrm{T} 3,4$ & 37 & 37.1 & 3.21 & & 2.55 & \\
\hline & & & & $(0.65-58.11)$ & & $(0.51-46.40)$ & \\
\hline
\end{tabular}

type patients was probably that the regulation of this signal pathway depends mainly on the binding of ligands such as AREG and EREG to HER receptors in KRAS wild-type individuals, which suggests that higher expression of ligands results in up-regulation of this pathway and leads to cancer cell proliferation and the likelihood of metastasis. On the other hand, it is believed that KRAS mutations can lead to dysregulation of this pathway and downstream signaling in the absence of liganddependent receptor activation [10], and this suggested that the expression of ligands cannot be the main regulating factor of this pathway in KRAS mutant individuals. In our data, the patients with high EREG expression showed longer survival time in KRAS mutant patients ( $p=0.045)$, in contrast to that in KRAS wildtype patients. However, since the numbers of patients with KRAS mutant were relatively small and the p-value was close to the significance level, this finding may not be reproducible, and probably needs to be validated.

Recently, several studies have reported that AREG (and EREG) expression was associated with the likelihood of liver metastasis in colorectal cancer, and suggested that it might play an important role in the development of liver metastasis [12,13]. In our study, a significant relationship was observed between EREG expression and patient survival, although all of the patients involved had liver metastases. This fact indicates that EREG and AREG expression is not only associated with the likelihood of metastasis but is also involved in tumor progression and biological malignancy. The association between these types of gene expression and survival time was also reported in other tumor types, such as non-small cell lung cancer [20] and oral squamous cell carcinoma [21]. These data suggested that EREG and AREG expression is not only a predictive biomarker for patients who have received anti-EGFR therapy, but is also a prognostic biomarker for a various types of cancer patients.

\section{Conclusions}

In summary, this is the first study which showed the correlation of AREG and EREG mRNA expression between primary and corresponding liver metastases. This study also showed the significance of EREG mRNA expression as prognostic marker in patients with KRAS wild-type. These data appear to support the fact that this HER family signaling pathway plays a very important role in tumor progression, and blocking this pathway is a reasonable strategy for the treatment of colorectal cancer.

\section{Acknowledgements and funding}

We thank Mr. Chris W. P. Reynolds of the International Medical Communications Center of Tokyo Medical University for his careful review of 
this manuscript. This study was financially supported by departmental funds from Tokyo Women's Medical University.

\section{Author details}

${ }^{1}$ Department of Chemotherapy and Palliative Care, Tokyo Women's Medical University, 8-1 Kawadacho, Shinjukuku, Tokyo, Japan. ${ }^{2}$ Department of Gastroenterological Surgery, Tokyo Women's Medical University, 8-1 Kawadacho, Shinjukuku, Tokyo, Japan.

\section{Authors' contributions}

HK conceived of the study, participated in the design of the study and drafted the manuscript. GN participated in the design of the study. YK carried out the DNA sequencing. AN performed the statistical analysis. YI participated in the design of the study. MY and $\mathrm{KH}$ helped to draft the manuscript. All authors read and approved the final manuscript.

\section{Competing interests}

The authors declare that they have no competing interests.

Received: 11 October 2011 Accepted: 13 March 2012

Published: 13 March 2012

\section{References}

1. Carpenter G, Cohen S: Epidermal growth factor. J Biol Chem 1990, 265(14):7709-7712.

2. Cunningham D, Humblet $Y$, Siena S, Khayat D, Bleiberg $H$, Santoro A, Bets D, Mueser M, Harstrick A, Verslype C, et al: Cetuximab monotherapy and cetuximab plus irinotecan in irinotecan-refractory metastatic colorectal cancer. N Engl J Med 2004, 351(4):337-345.

3. Van Cutsem E, Peeters M, Siena S, Humblet $Y$, Hendlisz A, Neyns B, Canon JL, Van Laethem JL, Maurel J, Richardson G, et al: Open-label phase III trial of panitumumab plus best supportive care compared with best supportive care alone in patients with chemotherapy-refractory metastatic colorectal cancer. J Clin Oncol 2007, 25(13):1658-1664.

4. Saltz LB, Meropol NJ, Loehrer PJ Sr, Needle MN, Kopit J, Mayer RJ: Phase II trial of cetuximab in patients with refractory colorectal cancer that expresses the epidermal growth factor receptor. J Clin Oncol 2004 , 22(7):1201-1208

5. Bokemeyer C, Bondarenko I, Makhson A, Hartmann JT, Aparicio J, de Braud F, Donea S, Ludwig H, Schuch G, Stroh C, et al: Fluorouracil, leucovorin, and oxaliplatin with and without cetuximab in the first-line treatment of metastatic colorectal cancer. J Clin Oncol 2009, 27(5):663-671.

6. Lievre A, Bachet JB, Boige V, Cayre A, Le Corre D, Buc E, Ychou M, Bouche $O$, Landi B, Louvet $C$, et al: KRAS mutations as an independent prognostic factor in patients with advanced colorectal cancer treated with cetuximab. J Clin Oncol 2008, 26(3):374-379.

7. Douillard JY, Siena S, Cassidy J, Tabernero J, Burkes R, Barugel M, Humblet Y, Bodoky G, Cunningham D, Jassem J, et al: Randomized, phase III trial of panitumumab with infusional fluorouracil, leucovorin, and oxaliplatin (FOLFOX4) versus FOLFOX4 alone as first-line treatment in patients with previously untreated metastatic colorectal cancer: the PRIME study. J Clin Oncol 2010, 28(31):4697-4705.

8. Allegra CJ, Jessup JM, Somerfield MR, Hamilton SR, Hammond EH, Hayes DF, McAllister PK, Morton RF, Schilsky RL: American Society of Clinical Oncology provisional clinical opinion: testing for KRAS gene mutations in patients with metastatic colorectal carcinoma to predict response to anti-epidermal growth factor receptor monoclonal antibody therapy. J Clin Oncol 2009, 27(12):2091-2096.

9. Inatomi $O$, Andoh A, Yagi Y, Bamba S, Tsujikawa T, Fujiyama Y: Regulation of amphiregulin and epiregulin expression in human colonic subepithelial myofibroblasts. Int J Mol Med 2006, 18(3):497-503.

10. Khambata-Ford S, Garrett CR, Meropol NJ, Basik M, Harbison CT, Wu S, Wong TW, Huang X, Takimoto CH, Godwin AK, et al: Expression of epiregulin and amphiregulin and K-ras mutation status predict disease control in metastatic colorectal cancer patients treated with cetuximab. J Clin Oncol 2007, 25(22):3230-3237.

11. Jacobs B, De Roock W, Piessevaux H, Van Oirbeek R, Biesmans B, De Schutter J, Fieuws S, Vandesompele J, Peeters M, Van Laethem JL, et al: Amphiregulin and epiregulin mRNA expression in primary tumors predicts outcome in metastatic colorectal cancer treated with cetuximab. J Clin Oncol 2009, 27(30):5068-5074.

12. Yamada M, Ichikawa Y, Yamagishi S, Momiyama N, Ota M, Fujii S, Tanaka K, Togo S, Ohki S, Shimada H: Amphiregulin is a promising prognostic marker for liver metastases of colorectal cancer. Clin Cancer Res 2008, 14(8):2351-2356

13. Watanabe T, Kobunai T, Yamamoto $Y$, Kanazawa T, Konishi T, Tanaka T, Matsuda K, Ishihara S, Nozawa K, Eshima K, et al: Prediction of liver metastasis after colorectal cancer using reverse transcription-polymerase chain reaction analysis of 10 genes. Eur J Cancer 2010, 46(11):2119-2126.

14. Kuramochi H, Hayashi K, Uchida K, Miyakura S, Shimizu D, Vallbohmer D, Park S, Danenberg KD, Takasaki K, Danenberg PV: Vascular endothelial growth factor messenger RNA expression level is preserved in liver metastases compared with corresponding primary colorectal cancer. Clin Cancer Res 2006, 12(1):29-33.

15. Kuramochi $\mathrm{H}$, Hayashi K, Nakajima G, Kamikozuru H, Yamamoto M, Danenberg KD, Danenberg PV: Epidermal growth factor receptor (EGFR) mRNA levels and protein expression levels in primary colorectal cancer and corresponding liver metastases. Cancer Chemother Pharmacol 2010, 65(5):825-831.

16. Kuramochi H, Hayashi K, Uchida K, Miyakura S, Shimizu D, Vallbohmer D, Park S, Danenberg KD, Takasaki K, Danenberg PV: 5-fluorouracil-related gene expression levels in primary colorectal cancer and corresponding liver metastasis. Int J Cancer 2006, 119(3):522-526.

17. Oliveras-Ferraros C, Vall-Llovera AM, Salip DC, Vazquez-Martin A, Cufi S, Queralt B, Martin-Castillo B, Brunet J, de Llorens R, Menendez JA: Evolution of the predictive markers amphiregulin and epiregulin mRNAs during long-term cetuximab treatment of KRAS wild-type tumor cells. Invest New Drugs 2012, 30(2):846-852.

18. Baker JB, Dutta D, Watson D, Maddala T, Munneke BM, Shak S, Rowinsky EK, Xu LA, Harbison $C T$, Clark EA, et al: Tumour gene expression predicts response to cetuximab in patients with KRAS wild-type metastatic colorectal cancer. Br J Cancer 2010, 104(3):488-495.

19. Jonker DJK, Harbison C: High epiregulin (EREG) gene expression plus Kras wild-type (WT) status as predictors of cetuximab benefit in the treatment of advanced colorectal cancer (ACRC): Results from NCIC CTG CO.17-A Phase III trial of cetuximab versus best supportive care (BSC). ASCO Annual Meeting 2009.

20. Zhang J, Iwanaga K, Choi KC, Wislez M, Raso MG, Wei W, Wistuba II, Kurie JM: Intratumoral epiregulin is a marker of advanced disease in non-small cell lung cancer patients and confers invasive properties on EGFR-mutant cells. Cancer Prev Res (Phila) 2008, 1(3):201-207.

21. Shigeishi H, Higashikawa K, Hiraoka M, Fujimoto S, Mitani Y, Ohta K, Takechi M, Kamata N: Expression of epiregulin, a novel epidermal growth factor ligand associated with prognosis in human oral squamous cell carcinomas. Oncol Rep 2008, 19(6):1557-1564.

\section{Pre-publication history}

The pre-publication history for this paper can be accessed here: http://www.biomedcentral.com/1471-2407/12/88/prepub

doi:10.1186/1471-2407-12-88

Cite this article as: Kuramochi et al:: Amphiregulin and Epiregulin mRNA expression in primary colorectal cancer and corresponding liver metastases. BMC Cancer 2012 12:88

\section{Submit your next manuscript to BioMed Central and take full advantage of:}

- Convenient online submission

- Thorough peer review

- No space constraints or color figure charges

- Immediate publication on acceptance

- Inclusion in PubMed, CAS, Scopus and Google Scholar

- Research which is freely available for redistribution 